\title{
Transcutaneous Electrical Nerve Stimulation versus Laser on Shoulder Functional Performance in Living Liver Donors
}

\author{
Ahmed NM Ahmed"1, Eman M Othman ${ }^{2}$, Hossam EM Soliman', Walid AI Abouelnaga ${ }^{1}$ \\ ${ }^{1}$ Department of Physical Therapy for Surgery, Faculty of Physical Therapy, Cairo University, Giza, Egypt \\ ${ }^{2}$ National Liver Institute, Menoufia University, Menoufia, Egypt \\ *Corresponding author: Ahmed NM Ahmed, Mobile: (+20) 01066428968 E-Mail: drahmednabil771 @ gmail.com
}

\begin{abstract}
Background: In living donor liver transplantation (LDLT), reported donors` mortality and morbidity were highly correlated with surgeon skills. Therefore, liver transplantation has become a distinct maneuver. In addition, minor liver surgeries complications include shoulder pain, pruritus, urinary retention, as well as major morbidity such as central venous catheter-induced thrombosis of brachial and/or subclavian vein, neuropraxia, foot drop also prolonged post-dural puncture headache.
\end{abstract}

Objective: to compare effectiveness of transcutaneous electrical nerve stimulation (TENS) versus low level laser therapy (LLLT) on pain and functional performance of liver donors' suffering from shoulder pain.

Patients and Methods: Thirty donors of both genders with ages ranging from 20 to 40 years old were selected from the Physical Therapy Outpatient Clinic of National Liver Institute, Menoufia University. They were randomly allocated into two equal groups: Group A included 15 donor patients who received 30 minutes TENS, 3 times per week, plus conventional physical therapy exercise program for 12 sessions along 4 weeks and group B that included 15 donor who received fifteen minutes LLLT 3 times per week plus conventional physical therapy exercise program for 12 sessions along 4 weeks.

Results: A significant improvement of VAS values post-treatment in both groups, also a significant SST benefits, plus improvement in shoulder flexion and abduction post-treatment compared to pre-values $(p<0.001)$ unless group A values that were superior to those of group B.

Conclusion: It could be concluded that both TENS as well LASER were effective in shoulder functional performance in donor` painful shoulders, but TENS was more effective.

Keywords: Living donor liver transplantation, Shoulder pain, TENS, LLLT.

\section{INTRODUCTION}

Liver has been classified as an internal organ interacts with all human` systems, so that individuals received or donated liver graft faces huge physiological changes ${ }^{(1)}$. Postoperative donors faces shoulder pain, foot drop also compartment syndrome, which might be explained due to intraoperative malpositioning. That is why must ensure that proper positioning is whole surgical team obligation including the anesthesiologist ${ }^{(2)}$. Commonly, liver donors` shoulder pain determined as an important musculoskeletal complication that requires multidisciplinary management including self-advice, analgesics and relative rest, as well access to physiotherapy (3). Donors' painful shoulders' physiotherapy protocols includes various modalities including cryotherapy, electrotherapy and mobilizing techniques in addition to therapeutic exercise training. Clinically, usual electrotherapy applications were ultrasound therapy, transcutaneous electrical nerve stimulation (TENS) as well as laser for painful donors shoulders ${ }^{(4)}$. In addition, TENS is non-invasive, analgesic technique that is claimed to have an effect. Worldwide TENS usage for various musculoskeletal painful disorders throughout health care is considered a common managing modality ${ }^{(5)}$. Besides, low level laser therapy
(LLLT) has been determined as effective physical modality for gaining analgesia also accelerates healing of numerous clinical disorders such as painful shoulders, which is explained based on biostimulation with light energy enhancing homeostasis level. LLLT is used in acute, chronic painful and inflammatory affections by very weak $(1-10 \mathrm{~mW})$ irradiation in special wavelength of $904 \mathrm{~nm}^{(6)}$.

The aim of the present study was to compare effectiveness of transcutaneous electrical nerve stimulation (TENS) versus low level laser therapy (LLLT) on pain and functional performance of liver donors' suffering from shoulder pain.

\section{PATIENTS AND METHODS}

Thirty liver donors' participants of both genders whose ages were 20- 40 years old. They were randomly selected from Physical Therapy Outpatient Clinic of National Liver Institute, Menoufia University. Then, they were allocated into two equal groups. Liver donors participants that were suffering from unilateral painful shoulder, had assigned their informed consent, if free of study exclusion criteria, which included cardiac and pulmonary disorders, diabetes mellitus or other 
orthopedic limitations, psychiatric and neurological disorders.

\section{Ethical consent:}

A single-blinded randomized controlled clinical that study was approved by Ethical Committee of Faculty of Physical Therapy, Cairo University. Research Ethics Committee and quality control approvals were held. Present study was explained in details and in plain terms to each participant before assigned their informed written consent. Quality control of screening, handling of data and verification of adherence to protocols were done on a regular basis by trial coordinator. This work has been carried out in accordance with The Code of Ethics of the World Medical Association (Declaration of Helsinki) for studies involving humans.

\section{Treatment:}

Visual Analogue Scale (VAS) was used to evaluate pain. Simple shoulder test (SST) was used to examine functional performance and Goniometry to assess affected shoulder flexion and abduction at beginning and by end of the study that extended for four weeks.

Group A: 15 liver donor participants that received thirty minutes TENS, 3 times per week, plus conventional physical therapy exercise for 12 sessions along 4 weeks.

Group B: 15 liver donor participants that received fifteen minutes LLLT 3 times per week, plus conventional physical therapy exercise for 12 sessions along 4 weeks (6).

Visual Analogue Scale (VAS) is considered as gold standard that is used particularly in pain-academic research. VAS consists of a $10 \mathrm{~cm}$ long horizontal line then single question in standardized wording: 'no pain' on left edge of the line, also 'worst imaginable pain' on the right line side, a mark was placed in corresponding to participants` pain intensity level ${ }^{(7)}$.

The shoulder simple test (SST) in brief, is valid, easy, short, reliable and self-report responsive questionnaire that consists of twelve questions with dichotomous response options of " $1=$ yes" or " $0=$ no". Each SST item is classified as function related pain (2 items), function/strength (7 items), and range of motion (3 items). SST total score ranges from zero (extreme physical limitations in function) to 12 (no functional limitation) simply, zero (worst function) and 100 (best function). Scoring is calculated as follows ("yes" responses repetitions/ number of answered items $) \times 100=$ $\%^{(8)}$.

Shoulder flexion and abduction were examined by Goniometry as an essential assessing musculoskeletal tool in practice era. It is used to determine any sort of physical dysfunction that guide treatment and generates evidence for treatment effectiveness. Universal goniometers (UG) were the most common goniometer tool used clinically due to its easily accessible, relatively inexpensive and portable ${ }^{(8)}$.

\section{Physical treatment- Group A/ TENS}

- Initial assessment using VAS, SST and shoulder flexion and abduction were taken after 4 weeks of study treatment protocol.

- Participant was placed in comfortable supine position.

- Affected shoulder' supraspinatus and posterior deltoid muscles were selected as treatment target, key maintaining correct shoulder alignment those providing stabilization. Therapeutic electrical stimulation to the supraspinatus and posterior deltoid muscles has been assigned to reduce shoulder pain, improve muscular force, and facilitate shoulder stability ${ }^{(\mathbf{1 0})}$.

- Four surface electrodes $(4 \times 4 \mathrm{~cm})$ were placed on targeted area in cross form then participant received TENS in conventional mode $(100 \mathrm{~Hz}, 120 \mu$ s and 30$40 \mathrm{~mA}$ ) for 20-30 minutes per session ${ }^{(4,11)}$.

\section{Physical treatment- Group B/ LLLT}

- Initial assessment using VAS, SST and shoulder flexion and abduction were taken after 4 weeks of study treatment protocol.

- Participant was placed in comfortable supported sitting position.

- Participant was wearing eye glasses to prevent retinae damage.

- LLLT (Ga As type 1-10 mW and $904 \mathrm{~nm}$ ), was irradiated on painful points minimum two-maximum four points every 3-4 minutes and maximum 15 minutes, in total ${ }^{(4)}$.

- The most painful points on deltoid and/or upper trapezius muscles ${ }^{(\mathbf{1 2})}$.

\section{Statistical analysis}

Descriptive analysis also unpaired t-test were conducted for comparing study groups demographics. Chi- squared was conducted for comparing of gender distribution in between groups. Normal data distribution was checked using Shapiro-Walk test for all variables. Levine's test for homogeneity of variances was carried out. In addition, unpaired t-test was carried out to compare VAS, SST, flexion and abduction mean values in between study groups. Paired t-test was conducted for comparing between pre- and post-treatment per group. Study level of significance was set at $\mathrm{p} \leq 0.05$, using statistical package for social studies (SPSS) version 25 for windows (IBM SPSS, Chicago, IL, USA). 


\section{RESULTS}

Table (1) showed study populations characteristics, there was no significant difference in between groups in age and gender distribution $(p>0.05)$.

Table (1): Basic characteristics of participants

\begin{tabular}{|c|c|c|c|}
\hline & Group A & Group B & \multirow{2}{*}{$\begin{array}{c}P- \\
\text { value }\end{array}$} \\
\hline & Mean \pm SD & Mean \pm SD & \\
\hline Age (years) & $29.73 \pm 5.2$ & $31.13 \pm 4.71$ & 0.44 \\
\hline Sex, n (\%) & & & \\
\hline Females & $5(33 \%)$ & $6(40 \%)$ & 0.7 \\
\hline Males & $10(67 \%)$ & $9(60 \%)$ & \\
\hline
\end{tabular}

$\mathrm{SD}$, standard deviation; $\mathrm{p}$-value, level of significance
Effect of treatment on VAS, SST, flexion and abduction:

- Within group comparison:

A significant diminished VAS values posttreatment in group A \& B compared to those pre-ones ( $\mathrm{p}$ $<0.001$ ) with percentages were $76.1 \%$ \& $44.3 \%$, respectively. Additionally, there was a significant improvement of SST, flexion and abduction posttreatment values in both groups compared to pre-values $(\mathrm{p}<0.001)$ with percentages of $140.44 \%, 26.32 \%$ and $37.1 \%$ respectively for Group A. While in group B, they were $68.25 \%, 14.08 \%$ and $20.68 \%$, respectively (Table 2).

\section{- Between groups' comparison:}

No significant differences in between groups' pretreatment $(p>0.05)$. Comparison in between groups posttreatment reported a significant improvement in VAS of group A compared to group B $(p<0.001)$ and a significant gain in SST, flexion and abduction of group A compared to group B $(\mathrm{p}<0.01)$ (Table 2$)$.

Table (2): Mean VAS, SST, flexion and abduction ROM pre and post treatment of the group A and B

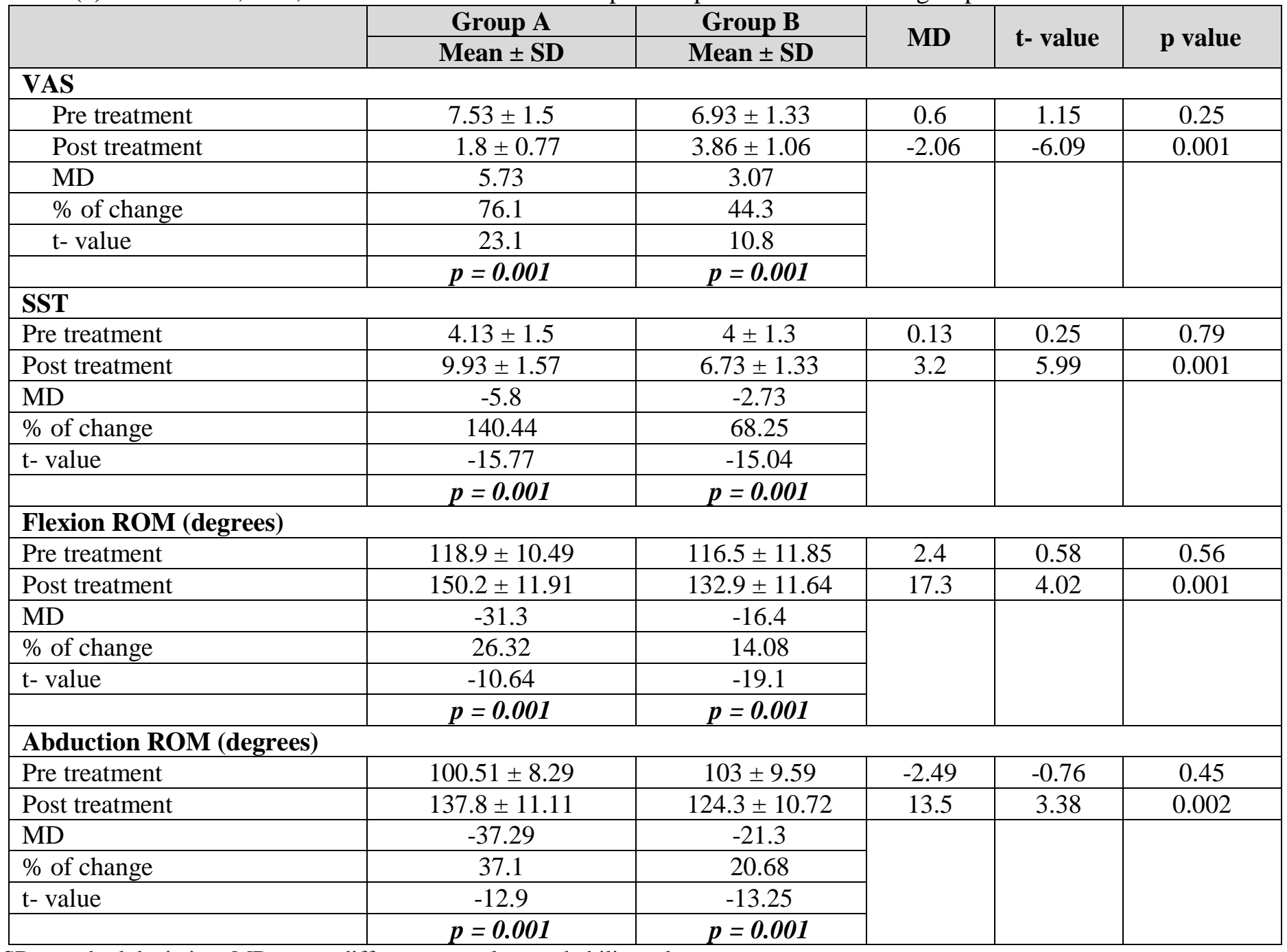

$\mathrm{SD}$, standard deviation; $\mathrm{MD}$, mean difference; $\mathrm{p}$-value, probability value 


\section{DISCUSSION}

Living donor liver transplantation has become an alternative therapeutic maneuver in end-stage liver lesions. Always, liver donors were healthy persons as well their safety remained as primary concern. The objective of our clinical trial was to compare effectiveness of TENS versus LLLT on pain and functional performance of liver donors' painful shoulder.

In LDLT, donors` mortality and morbidity were highly correlated with surgeon skills. Therefor LDLT has become a distinct maneuver where anesthetists have a crucial role ${ }^{(\mathbf{1 3})}$. In addition, minor liver surgeries complications includes shoulder pain, pruritus, urinary retention, and major morbidity such as central venous catheter-induced thrombosis of brachial and/or subclavian vein, neuropraxia, foot drop and prolonged post dural puncture headache ${ }^{(14)}$.

According to medical literature, predominantly, phrenic nerve arises unilaterally from the $3^{\text {rd }}, 4^{\text {th }}$ also $5^{\text {th }}$ cervical spinal roots then supply both shoulders as well diaphragm where providing regular diaphragmatic contractions during breathing. In particular, carbon dioxide-induced phrenic irritation leads to referred pain to fourth cervical dermatome and painful shoulder ${ }^{(\mathbf{1 5})}$.

Previous study conducted by Akpek et al. ${ }^{(16)}$ comprehensively enumerated anesthetic thirty donors` complications such as shoulder and neuropraxia representing $3.3 \%$ of their study populations, which explained to be due to overstretching during extensive arm retraction intraoperatively. Furthermore, Schumann et $\boldsymbol{a l} \mathbf{~}^{(\mathbf{1 7})}$ examined temporary donors' painful shoulder associated with diaphragmatic intraoperative irritation as well indwelling subdiaphragmatic surgical drains in nearby $\leq 75 \%$ of their series. They suggested that postoperative epidural analgesia alone was not sufficient to prevent such complications. In brief, liver donors` painful shoulders were explained due to intrinsic pathologies in its periarticular structures such as supraspinatus, bicipital and/or rotator cuff tendinitis, impingement syndromes or supraspinatus rupture, as well as subacromial bursitis, arthritis even frozen shoulder ...etc. where all of them restrict shoulder mobility in painful manner leading to disability ${ }^{(\mathbf{1 8})}$.

According to physical therapy shoulder rehabilitation guidelines, main goals could be enumerated in pain modulation, restoring muscular strength and maximizing dynamic function ${ }^{\left({ }^{19)}\right.}$. Most painful shoulder rehabilitation protocols include various modalities such as cryotherapy, electrotherapy, mobilization techniques and therapeutic exercising. Clinically, usual electrotherapy applications were ultrasound therapy, transcutaneous electrical nerve stimulation (TENS) as well as laser for painful donors`shoulders ${ }^{(5)}$.
Our clinical trial results recorded a significant VAS post-treatment gains in group A \& B compared to pre-ones $(\mathrm{p}<0.001)$ with percentages of $76.1 \%-44.3 \%$, respectively. Besides, there was significant improvement of SST, flexion and abduction post-treatment in both groups A \& B compared to pre-ones $(\mathrm{p}<0.001)$ with percentages in group A were $140.44 \%, 26.32 \%$ and $37.1 \%$, respectively. While, in group B they were $68.25 \%$, $14.08 \%$ and $20.68 \%$, respectively. Our results agree with the outcomes of Sluka ${ }^{(5)}$ represented twenty randomized clinical trials, two of them had examined cold pack and TENS, one clinical study evaluated therapeutic exercise therapy and another four clinical trials studied effectiveness of LLLT and could not find statistically significant difference on painful soft shoulders structures. In the same line, Arzu ${ }^{(4)}$ found statistically significant differences in pain intensity and shoulders mobility and its functional performance in response to TENS, LLLT and combination of both therapeutic modalities for painful shoulder management. Actually, they could improve shoulder mobility and its functional performance plus modulation of felt pain intensity even if applied separately.

Previous studies concerning TENS conducted by Bjordal et al. ${ }^{(20)}$, Brosseau et al. ${ }^{(21)}$, Rutjes et al. ${ }^{(22)}$, Johnson et al. ${ }^{(23)}$ and Bennett et al. ${ }^{(24)}$ recommended extensive TENS usage for acute as well as wide variety of chronic painful $(\geq$ three months' duration) musculoskeletal lesions aiming to modulate postoperative pain. It was considered effective mainly in acute orofacial pain, painful dental maneuvers, fractured ribs and acute low back or shoulder pain disorders. In addition, numerous experimental TENS studies carried out by Jain et al. ${ }^{(25)}$, Dabholkar et al. ${ }^{(26)}$, Hasan ${ }^{(27)}$ and Manoj et al. (28) reported that TENS was beneficial as an adjunct in management of type-1 complex regional pain syndrome, trigeminal neuralgia, labor low back pain, bruxismassociated masticatory pain, post-operative thoracic pain, cervical radiculopathy, sacroiliac joint dysfunction, fracture rib, diabetic neuropathy in addition to temporomandibular dysfunction, and painful shoulder.

On the other hand, cold laser recognized as noninvasive, nonionizing and monochromatic electromagnetic high concentrated light beam (LLLT) was widely used in various rheumatologic as well musculoskeletal disorders aiming for its analgesic, antiinflammatory and biostimulating effects. Moreover, LLLT was documented to induce cellular proliferation, collagen synthesis also protein synthesis, tissue reparation as well as wound healing and pain modulation through direct irradiation without any thermal response ${ }^{(\mathbf{2 9})}$. In the same line, LLLT was recommended to be combined with therapeutic exercise training than exercise training alone for pain management and functional benefits in rehabilitation of various musculoskeletal injuries. 
According to physical therapy evidence-based practice, numerous recommendations of LLLT widespread usage, especially in shoulder management, unless there were insufficient unclear evidences reported that LLLT contributes to pain modulations and improvement of functional limitations, particularly in shoulder disorders rehabilitation ${ }^{(3)}$. Furthermore, limited clinical studies had examined effectiveness of LLLT in shoulder rehabilitation. Bingöl et al. ${ }^{\left({ }^{(30)}\right.}$ has ensured no beneficial LLLT extra gains even if combined with exercise training compared to placebo.

This clinical study was limited to physical as well psychological participants' status those might affect trial evaluation and treatment measures`values.

\section{CONCLUSION}

It can be concluded that both TENS and LASER are effective physical therapy modalities for improving shoulder functional performance, but TENS is considered more effective.

\section{Financial support and sponsorship: Nil. Conflict of interest: Nil.}

\section{REFERENCES}

1. Murray K, Carithers R (2005): Practice guidelines: Evaluation of the patient for liver transplantation. Hepatology, 41: 1407-32.

2. Settmacher U, Theruvath T, Pascher A et al. (2004): Livingdonor liver transplantation European experiences. Nephrol Dial Transplant, 19 (4): 16-21.

3. Mitchell C, Adebajo A, Hay E et al. (2005): Shoulder pain: diagnosis and management in primary care. BMJ., 331: 1124-8.

4. Ozdincler A (2005): Effects of TENS and LEL on Pain and Functional Performance of Patients with Shoulder Pain. Journal of Medical Sciences, 5: 328-332.

5. Sluka K, Walsh D (2009): Transcutaneous electrical nerve stimulation: Basic science mechanisms and clinical effectiveness. J Pain, 4: 109-21.

6. Green S, Buchbinder R, Hetrick S (2003): Physiotherapy interventions for shoulder pain. Cochrane database Syst Rev., 2: $42-48$.

7. Delgado D, Lambert B, Boutris $\mathrm{N}$ et al. (2018): Validation of Digital Visual Analog Scale Pain Scoring With a Traditional Paper-based Visual Analog Scale in Adults. Orthop Surg Glob Res., 2 (3): 88-93.

8. Roy J, Macdermid J, Faber $\mathbf{K}$ et al. (2010): The simple shoulder test is responsive in assessing change following shoulder arthroplasty. J Orthop Sports Phys Ther., 40 (7): 413-421.

9. Russell T, Jull G, Wooton R (2003): Can internet be used as a medium to evaluate knee angle? Manual Therapy, 8 (4): 242-246.

10. Koyuncu E, Nakipoglu-Yuzer G, Dogan A et al. (2010): The effectiveness of functional electrical stimulation for the treatment of shoulder subluxation and shoulder pain in hemiplegic patients: a randomized controlled trial. Disabil Rehabil., 32: 560-6.

11. Pushpa R, Vasantha K, Tarun G et al. (2020): Effect of transcutaneous electrical nerve stimulation therapy on pain and functional disability level among patients with rotator cuff disease- a randomized controlled tria. Int J Physiother., 7 (1): 7 13.
12. Schneider R, Prentice W (2001): Rehabilitation of the shoulder." In orthopaedic rehabilitation, (Eds. Nickel, v.L. and M.J. Botte). $2^{\text {nd }}$ Edn., Churchill living stone, New York, Pp: 747763.

13. Nadalin S, Malago M, Radtke A et al. (2007): Current trends in live liver donation. Transpl Int., 20: 312-330.

14. Ozkardeslera D, Ozzeybeka E, Alaygutb T et al. (2008): Anesthesia-Related Complications in Living Liver Donors: The Experience from One Center and the Reporting of One Death. American Journal of Transplantation, 8 (10): 2106-10.

15. Tsai H, Chen Y, Ho C et al. (2011): Maneuvers to Decrease Laparoscopy-Induced Shoulder and Upper Abdominal Pain: A Randomized Controlled Study. Arch Surg., 146 (12): 1360-1366.

16. Akpek E, Arslan G, Erkaya C et al. (2003): Anesthetic risks for donors in living-related liver transplantation: Analysis of 30 cases. Transpl Int., 16: 584-588.

17. Schumann R, Zabala L, Angelis M et al. (2004): Altered hematologic profiles donor right hepatectomy and implications for perioperative analgesic management. Liver Transpl., 10: 363368.

18. Gursel Y, Ulus Y, Bilgic A (2004): Adding ultrasound in the management of soft tissue disorders of the shoulder: A randomized placebo-controlled trial. Phys Ther., 84: 336-343.

19. Bonafede R, Bennet $\mathbf{R}$ (2016): Shoulder pain: Guidelines to diagnosis and management. Postgrad Med., 82: 185-193.

20. Bjordal J, Johnson M, Ljunggreen A (2003): Transcutaneous electrical nerve stimulation (TENS) can reduce postoperative analgesic consumption. A meta-analysis with assessment of optimal treatment parameters for postoperative pain. Eur J Pain, 7 (2): 181-88.

21. Brosseau L, Judd M, Marchand S et al. (2003): Transcutaneous electrical nerve stimulation (TENS) for the treatment of rheumatoid arthritis in the hand. Cochrane Database Syst Rev., 3: 53-58.

22. Rutjes A, Nuesch E, Sterchi $\mathbf{R}$ et al. (2009): Transcutaneous electrostimulation for osteoarthritis of the knee. Cochrane Database of Systematic Reviews, 4: 96-102.

23. Johnson M, Oxberry S, Simpson K (2010): Transcutaneous Electrical Nerve Stimulation (TENS) and acupuncture for acute pain, in Acute Pain. In: Macintyre P., Walker S., Rowbotham D., editors. Clinical Pain Management. Second Edition. London: Hodder Arnold. Pp: 271-90.

24. Bennett M, Hughes N, Johnson M (2011): Methodological quality in randomised controlled trials of transcutaneous electric nerve stimulation for pain: low fidelity may explain negative findings. Pain, 156 (2): 1226-32.

25. Jain M, Sharma N, Kalra S (2011): To compare the effects of high and low frequency transcutaneous electrical nerve stimulation on acupuncture points in experimental pain threshold. Indian J Physiother Occup Ther., 5: 111-4.

26. Dabholkar T, Hutoxi W (2009): Effect of auricular transcutaneous electrical nerve stimulation on experimental pain threshold." Indian J Physiother Occup Ther., 3: 95-100.

27. Hasan S (2013): Comparative Study: Analgesic effect of AlTENS in variation of treatment time on experimentally induced ischaemic pain in healthy young adult" Indian J Physiother Occup Ther., 7: 255-60.

28. Manoj M, Kaur J (2011): Effect of unilateral and bilateral auricular acupuncture like TENS on Pain Threshold. Indian J Physiother Occup Ther., 5: 101-7 .

29. Dogan S, Saime A, Evcik D (2010): The effectiveness of low laser therapy in subacromial impingement syndrome: a randomized placebo controlled double-blind prospective study. Clinics (Sao Paulo), 65 (10): 1019-1022.

30. Bingöl U, Alatan L, Yurtkuran M (2005): Low-power laser treatment for shoulder pain. Photomed Laser Surg., 23: 459-64. 